\title{
High-Dimensional Clustering Method for High Performance Data Mining*
}

\author{
Jae-Woo Chang and Hyun-Jo Lee \\ Research Center of Industrial Technology \\ Dept. of Computer Engineering, Chonbuk National University, \\ Chonju, Chonbuk, 561-756, South Korea \\ jwchang@chonbuk.ac.kr, hjlee@dblab.chonbuk.ac.kr
}

\begin{abstract}
Many clustering methods are not suitable as high-dimensional ones because of the so-called 'curse of dimensionality' and the limitation of available memory. In this paper, we propose a new high-dimensional clustering method for the high performance data mining. The proposed high-dimensional clustering method provides efficient cell creation and cell insertion algorithms using a space-partitioning technique, as well as makes use of a filtering-based index structure using an approximation technique. In addition, we compare the performance of our high-dimensional clustering method with the CLIQUE method which is well known as an efficient clustering method for highdimensional data. The experimental results show that our high-dimensional clustering method achieves better performance on cluster construction time and retrieval time than the CLIQUE.
\end{abstract}

Keywords: High-Dimensional Clustering, Data Mining.

\section{Introduction}

Data mining is concerned with the extraction of interesting knowledge from a large amount data, i.e. rules, regularities, patterns, constraints. Clustering, one of the most important research topics in data mining, is the process of grouping data into classes or clusters, in such a way that objects within a cluster have high similarity to one another, but are very dissimilar to objects in other clusters [1]. The existing clustering methods have a critical drawback that they do not work well for clustering highdimensional data because their retrieval performance is generally degraded as the number of dimension increases. In this paper, we propose an efficient highdimensional clustering method for the high performance data mining. Our highdimensional clustering method provides a cell creation algorithm to make cells by splitting each dimension into a set of partitions, and provides a cell insertion algorithm to construct clusters as cells with more density than a given threshold and insert them into an index structure. By using an approximation technique, we also propose a new filtering-based index structure to have fast accesses to the clusters.

\footnotetext{
This work is financially supported by the Ministry of Education and Human Resources Development (MOE), the Ministry of Commerce, Industry and Energy (MOCIE) and the Ministry of Labor (MOLAB) though the fostering project of the Lab of Excellency.
} 
The rest of this paper is organized as follows. The next section discusses related work on high-dimensional clustering methods. In Section 3, we propose a new highdimensional clustering method. In Section 4, we analyze the performances of our high-dimensional clustering method. Finally, we draw our conclusion in Section 5.

\section{Related Work}

The existing high-dimensional clustering methods can be roughly classified into two groups, such as grid-based and partitioning [2]. CLIQUE[3] and MAFIA[4] belong to the grid-based approach while PROCLUS[5], FINDIT[6], and DOC[7] belong to the partitioning approach. In this section, we discuss the typical clustering methods for each approach. First, CLIQUE(CLustering In QUEst) was proposed as a densitybased clustering method. CLIQUE automatically finds subspaces(grids) with highdensity clusters. CLIQUE produces identical results irrespective of the order in which input records are presented, and it does not presume any canonical distribution of input data. Input parameters are the size of the grid and a global density threshold for clusters. Next, PROCLUS was the first top-down clustering method while CLIQUE scales linearly with the number of input records and it has good scalability as the number of dimensions in the data. PROCLUS samples the data, then selects a set of $\mathrm{k}$ medoids, and iteratively improves the clustering. PROCLUS is biased toward clusters that are hyper-spherical in space. While cluster may be found in differ subspaces, the subspaces must be of similar sizes since the user must input the average number of dimensions for the clusters.

\section{An Efficient High-Dimensional Clustering Method}

Since the existing clustering methods assume that a data set is resident in main memory, they are not efficient for handling large amounts of data. As the dimensionality of data is increased, the number of cells increases exponentially, thus causing the dramatic performance degradation. To remedy that effect, we propose an efficient high-dimensional clustering method for the high performance data mining. The overall architecture of our clustering method is shown in Figure 1.

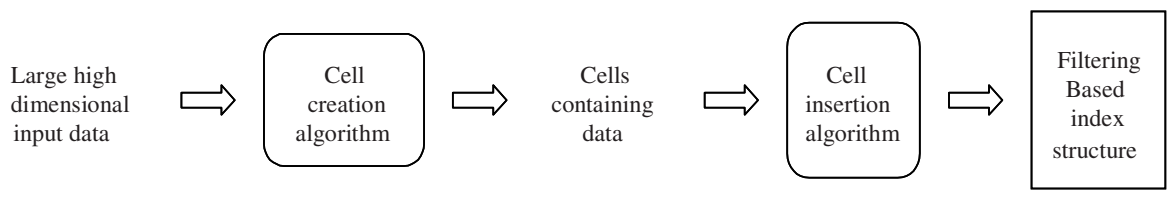

Fig. 1. Overall architecture of our clustering method

\subsection{Cell Creation Algorithm}

Our cell creation algorithm makes cells by splitting each dimension into a group of sections using a split index. Density based split index is used for creating split sections and is efficient for splitting multi-group data. Our cell creation algorithm first finds the optimal split section by repeatedly examining a value between the maximum 
and the minimum in each dimension. The split index value is calculated by Eq. (1) before splitting and Eq. (2) after splitting. Using Eq. (1), we can determine the split index value for a data set $S$ in three steps: i) divide $S$ into $C$ classes, ii) calculate the square value of the relative density $\left(\mathrm{P}_{\mathrm{j}}\right)$ of each class, and iii) subtract from one all the square values of the densities of $\mathrm{C}$ classes. Using Eq. (2), we compute a split index value for $S$ after $S$ is divided into $S_{1}$ and $S_{2}$. Here the number of data in $S$ is $n$ and the number of data belonging to $S_{1}\left(S_{2}\right)$ is $n_{1}\left(n_{2}\right)$. If the split index value is larger than the previous value before splitting, we actually divide $S$ into $S_{1}$ and $S_{2}$. Otherwise, we stop splitting. Secondly, our cell creation algorithm creates cells being made by the optimal split sections for $\mathrm{n}$-dimensional data. As a result, our cell creation algorithm creates fewer cells than the existing clustering methods using equivalent intervals.

$$
\begin{gathered}
\operatorname{Split}(S)=1-\sum_{j=1}^{C} P_{j}{ }^{2} \\
\operatorname{Split}(S)=\frac{n_{1}}{n} \operatorname{Split}\left(S_{1}\right)+\frac{n_{2}}{n} \operatorname{Split}\left(S_{2}\right)
\end{gathered}
$$

If a data set has $\mathrm{n}$ dimensions and the number of the initial split sections in each dimension is $\mathrm{m}$, the conventional cell creation algorithms make mn cells, but our cell creation algorithm makes only $\mathrm{K} 1 * \mathrm{~K} 2 * \ldots * \mathrm{Kn}$ cells $(1 \leq \mathrm{K} 1, \mathrm{~K} 2, . ., \mathrm{Kn} \leq \mathrm{m})$.

\subsection{Cell Insertion Algorithm}

For cell insertion, we first obtain the cells created using the cell creation algorithm. Secondly, we construct clusters as the cells with more density than a given cell threshold and store them into a cluster information file. A record in the cluster information file consists of a cluster id and the number of data belonging to the cluster. The cluster information file for large, high-dimensional data is too large to reside in memory. Thirdly, we calculate the frequency of a section in all dimensions whose frequency is greater than a given section threshold. Finally, we set to ' 1 ' the bits corresponding to the sections with a high frequency in an approximation information file. We set to ' 0 ' the other bits for the remainder sections. We calculate the frequency of data in a cell. Finally, the cell threshold and the section threshold are shown in Eq. (3).

Section threshold $=\left(\begin{array}{l}\lambda=\frac{N R \times F}{N I} \\ N I: \text { the number of input data } \\ N R: \text { the number of sections per dimension } \\ F: \text { minimum section frequency being regarded as '1' }\end{array}\right.$

Cell threshold $(\tau)$ : positive integer

\subsection{Filtering-Based Index Structure}

When the number of the created cells is very large due to large and high-dimensional data, it may take much time to answer users' queries. In order to reduce time to 
respond to the queries, it is possible to construct a new filtering-based index scheme using the approximation information file. Figure 2 shows an example of a filteringbased index scheme containing both the approximation information file and cluster information file, assuming two-dimensional data. Let assume that $\mathrm{K}$ clusters are created by our cell-based clustering method and the numbers of split sections in $\mathrm{X}$ axis and $\mathrm{Y}$ axis are $\mathrm{m}$ and $\mathrm{n}$, respectively. The following equation, Eq.(4), shows the retrieval times $(\mathrm{C})$ when the approximation information file is used and when it is not used. We assume that is an average filtering ratio in the approximation information file. $\mathrm{D}$ is the number of dimensions of input data. $\mathrm{P}$ is the number of records per page. $\mathrm{R}$ is the average number of records in each dimension. When the approximation information file is used, the retrieval time decreases as decreases. For high-dimension data, our two-level index scheme using the approximation information file is an efficient scheme because the $\mathrm{K}$ value increases exponentially in proportion to dimension D. The size of the approximation information file is dependent on both the number of dimensions of data and the numbers of split sections in each dimension. Because we deal with high-dimensional data, we keep the approximation information file in disk, rather than in memory.

i) Retrieval time without the use of an approximation information file $C=\lceil K / P\rceil / 2$ (Disk I/O accesses)

ii) Retrieval time with the use of an approximation information file

$$
C=\lceil(D * R) / P\rceil * \alpha+(1-\alpha)\lceil K / P\rceil / 2 \text { (Disk I/O accesses) }
$$

Figure 2 shows our filtering-based index scheme used to answer a query when a cell threshold and a section threshold are 1, respectively. For a query Q1, we determine 0.6 in $\mathrm{X}$ axis as the third section and 0.8 in $\mathrm{Y}$ axis as the fourth section. In the approximation information file, the value for the third section in $\mathrm{X}$ axis is ' 1 ' and the value for the 4-th section in Y axis is '0'. Because one of sections' values is ' 0 ', Q1 can be discarded without searching the corresponding cluster information file. For a query Q2, the value of 0.55 in $\mathrm{X}$ axis and the value of 0.7 in $\mathrm{Y}$ axis belong to the third section, respectively. Because the third bit for $\mathrm{X}$ axis and the third bit for $\mathrm{Y}$ axis have ' 1 ' in the approximation information file, we calculate a cell number and obtain its cell

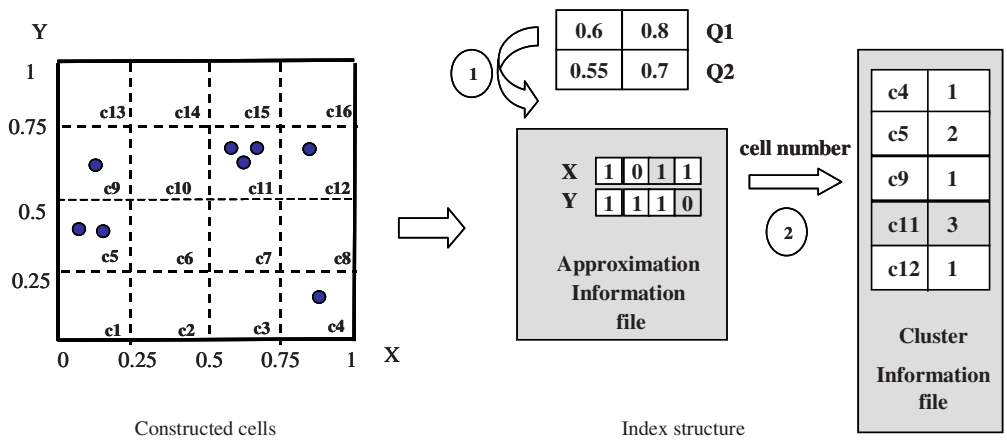

Fig. 2. Filtering-based index scheme 
frequency by accessing the cluster information file. As a result, we obtain a cell number 11 and its frequency 3 for Q2.

\section{Performance Analysis}

For our performance analysis, we implemented our clustering method on Linux server with dual processors. We make use of one million data (16-dimensional one) created by Synthetic Data Generation Code for Classification in IBM Quest Data Mining Project [8]. A record in our experiment is composed of both numeric type attributes, like salary, commission, age, hvalue, hyears, loan, tax, interest, cyear, balance, and categorical type attributes, like level, zipcode, area, children, ctype, job. The factors of our performance analysis are cluster construction time, precision, and retrieval time. We compare our cell-based clustering method (CBCM) with the CLIQUE method, which is one of the most efficient high-dimensional clustering methods. For our experiment, we make use of three data sets, one with random distribution, one with standard normal distribution (variation=1), and one with normal distribution of

Table 1. Methods used for performance comparison (MI:Maximal Interval)

\begin{tabular}{l|l}
\hline Methods & Description \\
\hline CBCM-5R & CBCM for data set with random distribution(MI =5) \\
CLIQUE-5R & CLIQUE for data set with random distribution (MI=5) \\
\hline CBCM-10R & CBCM for data set with random distribution (MI=10) \\
CLIQUE-10R & CLIQUE for data set with random distribution (MI=10) \\
\hline CBCM-5SND & CBCM with standard normal distribution (MI=5) \\
CLIQUE-5SND & CLIQUE with standard normal distribution (MI=5) \\
\hline CBCM-10SND & CBCM with standard normal distribution (MI=10) \\
CLIQUE-10SND & CLIQUE with standard normal distribution (MI=10) \\
\hline CBCM-5ND $(0.5)$ & CBCM with normal distribution of variation 0.5 (MI=5) \\
CLIQUE-5ND $(0.5)$ & CLIQUE with normal dist. of variation 0.5 (MI=5) \\
\hline CBCM-10ND $(0.5)$ & CBCM with normal distribution of variation $0.5(\mathrm{MI}=10)$ \\
CLIQUE-10ND $(0.5)$ & CLIQUE with normal dist. of variation 0.5 (MI=10) \\
\hline
\end{tabular}
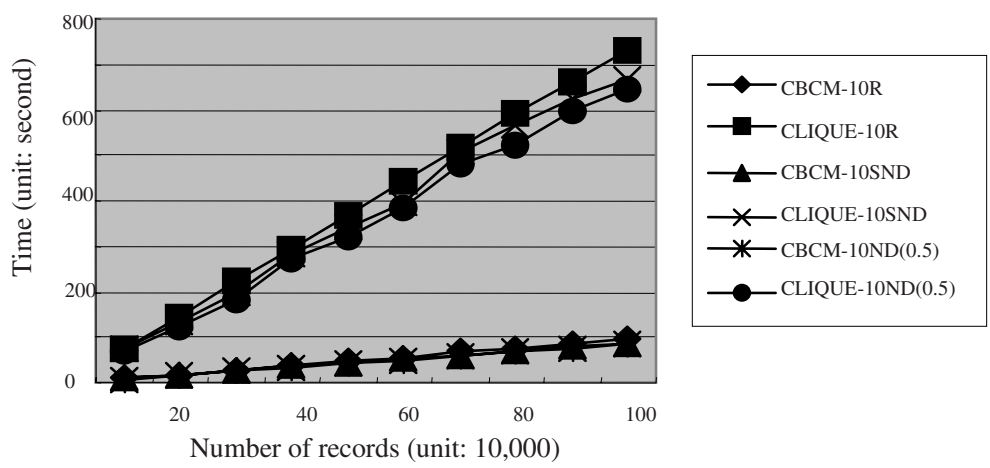

Fig. 3. Cluster Construction Time 
variation 0.5 . We also use 5 and 10 for the interval of numeric attributes. Table 1 shows methods used for performance comparison in our experiment.

Figure 3 shows the cluster construction time when the interval of numeric attributes equals 10 . It is shown that the cluster construction time increases linearly in proportion to the amount of data. This result is applicable to large amounts of data. The experimental result shows that the CLIQUE requires about 700 seconds for one million items of data, while our CBCM needs only 100 seconds. Because our method creates smaller number of cells than the CLIQUE, our CBCM method leads to $85 \%$ decrease in cluster construction time. The experimental result with the maximal interval $(\mathrm{MI})=5$ is similar to that with $\mathrm{MI}=10$.

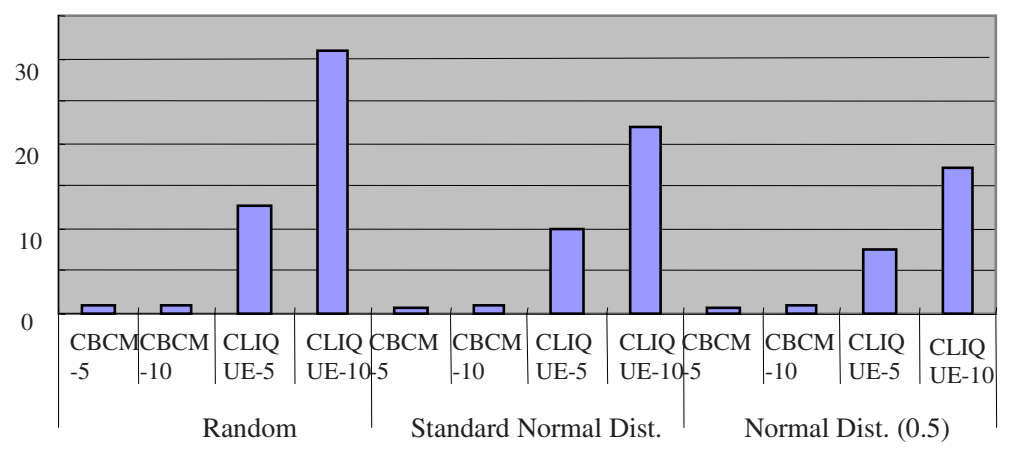

Fig. 4. Retrieval Time

Figure 4 shows average retrieval time for a given user query after clusters were constructed. When the interval of numeric attributes equals 10, the CLIQUE needs about 17-32 seconds, while our CBCM needs about 2 seconds. When the interval equals 5, the CLIQUE and our CBCM need about 8-13 seconds and 1 second, respectively. It is shown that our $\mathrm{CBCM}$ is much better on retrieval performance than the CLIQUE. This is because our method creates a small number of cells by using our cell creation algorithm, and achieves good filtering effect by using the approximation information file. It is also shown that the CLIQUE and our CMCM require long retrieval time when using a data set with random distribution, compared with normal distribution of variation 0.5 . This is because as the variation of a data set decreases, the number of clusters decreases, leading to better retrieval performance.

Figure 5 shows the precision of the CLIQUE and that of our CBCM, assuming that the section threshold is assumed to be 0 . The result shows that the CLIQUE achieves about $95 \%$ precision when the interval equals 10 , and it achieves about $92 \%$ precision when the interval equals 5. Meanwhile, our CBCM achieve over $90 \%$ precision when the interval of numeric attributes equals 10 while it achieves about $80 \%$ precision when the interval equals 5 . This is because the precision decreases as the number of clusters constructed increases.

Because both retrieval time and precision have a trade-off, we estimate a measure used to combine retrieval time and precision. To do this, we define a system efficiency measure in Eq. (5). Here $\mathrm{E}_{\mathrm{MD}}$ is the system efficiency of methods (MD) shown in Table 1 and $\mathrm{W}_{\mathrm{p}}$ and $\mathrm{W}_{\mathrm{t}}$ are the weight of precision and that of retrieval time, 


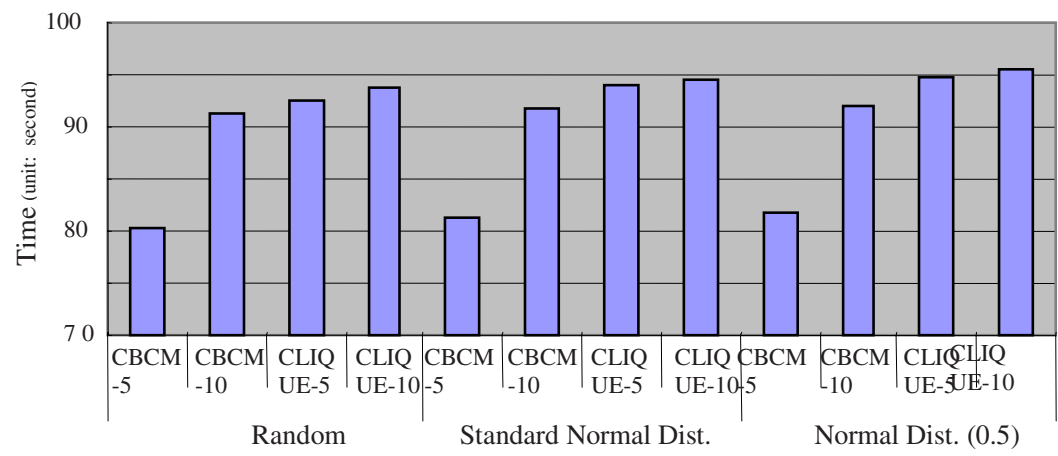

Fig. 5. Precision

respectively. $\mathrm{P}_{\mathrm{MD}}$ and $\mathrm{T}_{\mathrm{MD}}$ are the precision and the retrieval time of the methods (MD). $\mathrm{P}_{\mathrm{MAX}}$ and $\mathrm{T}_{\mathrm{MIN}}$ are the maximum precision and the minimum retrieval time, respectively, for all methods.

$$
E_{M D}=W_{p} \bullet \frac{P_{M D}}{P_{M A X}}+W_{t} \bullet \frac{1}{T_{M A X} / T_{M I N}}
$$

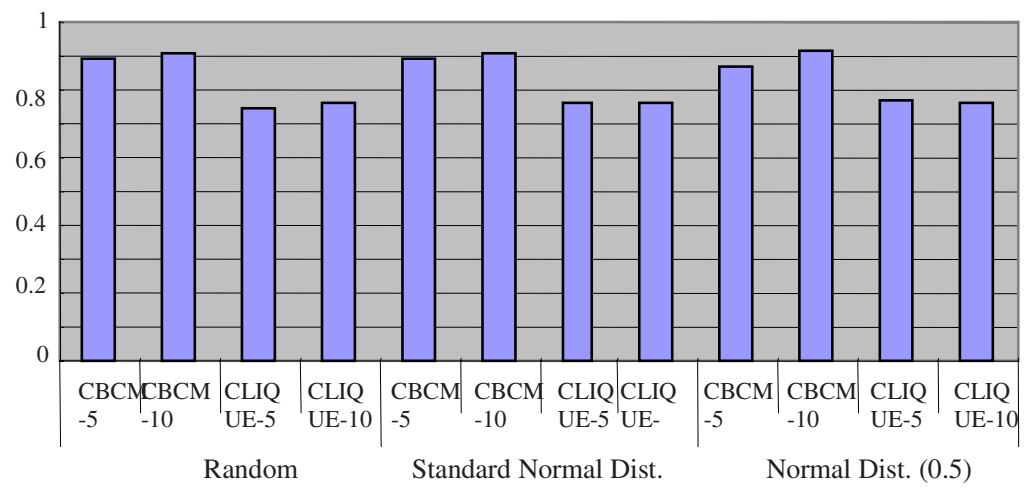

Fig. 6. System efficiency

Figure 6 depicts the performance results of methods in terms of their system efficiency. The importance of the precision and that of the retrieval time depend on an application situation, but the precision is more important than the retrieval time in general. Thus, it is reasonable that we should set the weight of the precision to be three times greater than that of retrieval time, i.e., $\mathrm{W}_{\mathrm{p}}=0.75, \mathrm{~W}_{\mathrm{t}}=0.25$. The performance result shows that our CBCM outperforms the CLIQUE with respect to the system efficiency, regardless of the data distribution of the data sets. Especially, the performance of our CBCM with $\mathrm{MI}=10$ is the best. 


\section{Conclusion}

The conventional clustering methods are not efficient for large, high-dimensional data. In order to overcome the difficulty, we proposed a new efficient clustering method with two features. The first one allows us to create the small number of cells for large, high-dimensional data. To do this, we calculate a section of each dimension through split index and create cells according to the overlapped area of each fixed section. The second one allows us to apply an approximation technique to our clustering method for the high performance data clustering. For this, we use a twolevel index structure which consists of both an approximation information file and a cluster information file. For performance analysis, we compare our high-dimensional clustering method with the CLIQUE method. The performance analysis results show that our clustering method shows slightly lower precision, but it achieves good performance on retrieval time as well as cluster construction time. Finally, our highdimensional clustering method shows a good performance on system efficiency which is a measure to combine both precision and retrieval time. As future work, it is required to study on the parallelism of our high-dimensional clustering method in order to achieve higher performance on retrieval.

\section{References}

1. Han, J., Kamber, M.: Data Mining: Concepts and Techniques. Morgan Kaufmann (2000).

2. Gan, G., Wu, J.: Subspace Clustering for High Dimensional Categorical Data. ACM SIGKDD Explorations networks, Vol. 6, Issue 2 (2004) 87-94.

3. Agrawal, R., Gehrke, J., Gunopulos, D., Raghavan, P.: Automatic Subspace Clustering of High Dimensional Data Mining Applications. Proc. of ACM SIGMOD (1998) 94-105.

4. Nagesh, H., Goil, S., Choudhary, A.: A Scalable Parallel Subspace Clustering Algorithm for Massive data Sets. Proc. of Int. Conf. on parallel Processing (2000) 477-486.

5. Aggrawal, C., Wolf, J., Yu, P., Procopiuc, C., Park, J.: Fast Algorithms for Projected Clustering. Proc. of ACM SIGMOD (1999) 61-72.

6. Woo, K., Lee, J.: FINDIT: A Fast and Intelligent Subspace Clustering Algorithm using Dimension Voting. PhD thesis, Korea Advanced Institute of Sci.\&Tech, Dept. of CS (2002).

7. Procopiuc, C., Jones, M., Agrawal, P., Murali, T.: A More Carlo Algorithm for Fast Projective Clustering. Proc. of ACM SIGMOD (2002) 418-427.

8. http://www.almaden.ibm.com/cs/quest 